\title{
Detection of Arrhythmia using Neural Network
}

\author{
Saumendra Kumar Mohapatra, Hemanta Kumar Palo, Mihir Narayan Mohanty \\ Electronics \& Communication Engineering, \\ ITER, Siksha 'O' Anusandhan Deemed to be University, Bhubaneswar, India \\ \{saumendramohapatra, hematapalo,mihirmohanty\}@soauniversity.ac.in
}

\begin{abstract}
There is an increase in cardio logical patients all over the world due to change in modern life style. It forces the medical researchers to search for smart devices that can diagnosis and predict the onset of cardiac problem before it is too late. This motivates the authors to predict Arrhythmia that can help both the patients and the medical practitioners for better healthcare services. The proposed method uses the frequency domain information which can represent the ECG signals of Arrhythmia patients better. Features representing the MITBIH Arrhythmia are extracted using the efficient Short Time Fourier Transform and the Wavelet transform. A comparison of these features is made with that of normal human being using Neural Network based classifier. Wavelet based features has shown an improvement of Accuracy over that of STFT features in classifying Arrhythmia as our results reveal. A Mean Square Error (MSE) of with wavelet transform has validated our results.
\end{abstract}

Keyword-Feature Extraction; Short Time Fourier Transform; Wavelet Transform; Classification; Neural Network

\section{INTRODUCTION}

$\mathrm{T}$ HE WORLD is fast expanding day by day that leads to rapid change in human lifestyle. It affects the human being both physically and psychologically. People are demanding more logical, compact, cost effective and accessible devices that can take care and guide their health regularly at ease. This has made the healthcare domain both burdening and competitive. Among many fatal diseases, the heart care is one of the mostly sought biomedical fields of research today. The WHO (World Health Organization) has reported around 17 million deaths only because of heart attack [1]. Most of the cardiovascular problems occur due to age, angina, high cholesterol levels, diabetes, diet, genetics, hypertension, smoking, HIV, work stress etc. Due to many reasons, it is very difficult to care and maintain a healthy heart always that makes the research challenging. Search for an efficient diagnosis and monitoring system that can predict accurately heart ailment has been ever increasing. It desires the automation of medical detection system that benefits the society struggling with heart related issues [2]. An effective diagnosis machine requires a signal that represents the heart disease accurately. The Electrocardiograms (ECG) has been a vital non-intrusive apparatus that provides the desired and dependable signal to the cardiologists as well as medical researchers. It has been a tool for analysis of different cardiovascular arrhythmias appearing briefly during routine check of a cardiac patient [3].
For the diagnosis and monitoring to be effective, the system desired equally discriminative features that can represent the ECG signal adequately. There have been many reliable features explored earlier by biomedical researchers in diagnosis of Arrhythmia patients using ECG. Most of these features are: temporal intervals [4], morphological features [5], statistical features [6], wavelet transform [7] etc. The ECG signal is generally described by five prominent peaks $\mathrm{P}, \mathrm{Q}, \mathrm{R}, \mathrm{S}$ and $\mathrm{T}$ as given in Fig.1.

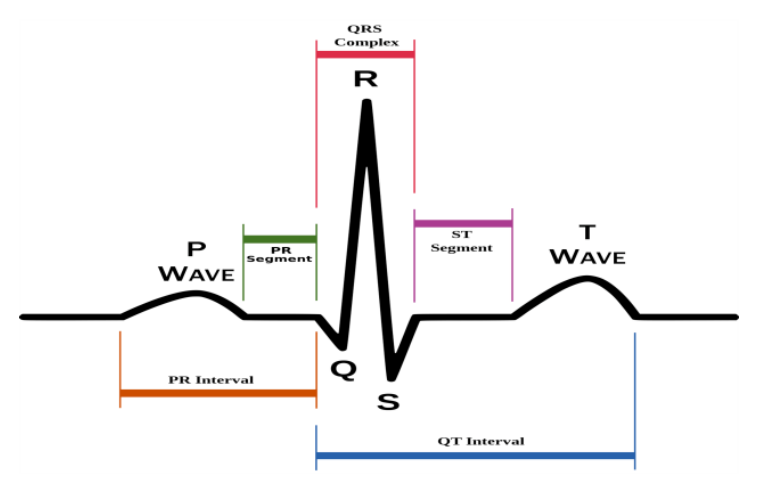

Fig.1. Five Peaks of a normal ECG Signal

The QRS envelope plays a major role in detection and analysis of ECG signal automatically. Wavelet transform (WT) provides the signal compression similar to STFT exclusively and can be utilized efficiently for normal or abnormal heart rhythm observation [8-9]. However, the features must be capable to simulate the pattern recognition tool for effective classification of the disease. Neural Networks (NN) have been serving as the powerful tools for data modeling in the past in the field of speech, image and bio-medical engineering. These information processing technique can capture complex input and output relation better because of their resemblance to human brain. These networks can answer those problems that have no algorithmic solutions. Among a host of NNs used in pattern identification the Multilayer Perceptron (MLP) is most popular and simple. It uses the hyperplanes for data space division which is the most natural approach based on the fundamental simplicity of lines and is intuitively appealing in our case. This has been used in this work for classification of the patients suffering from Arrhythmia with the chosen feature sets of ECG. 
The rest of the sections are organized as follows. The proposed feature extraction techniques and Classification techniques have been briefed in section-II. This part also describes the dataset implemented in this experiment. The result section has been described in section-III. Section-IV concludes the work with necessary feature directions.

\section{METHODOLOGY}

Data Preparation: The MIT-BIH Arrhythmia Database has been used in our experiment. In this, ECG signals are exported with various formats such as .mat, .dat, .csv etc. The .mat files are chosen from the data base for the desired feature extraction using MATLAB. To test the process in a genuine situation, the genuine Clinical ECG signals and the $\mathrm{BW}$ signals were considered in the simulation. The BW signals are procured from the MIT-BIH noise stress database whereas the real life clinical signals have been procured from the Arrhythmia database. The recording of the signals have been done with $360 \mathrm{~Hz}$ over $5 \mathrm{mV}$ range having 11 bit resolution. Fig. 2 describes the proposed methodology

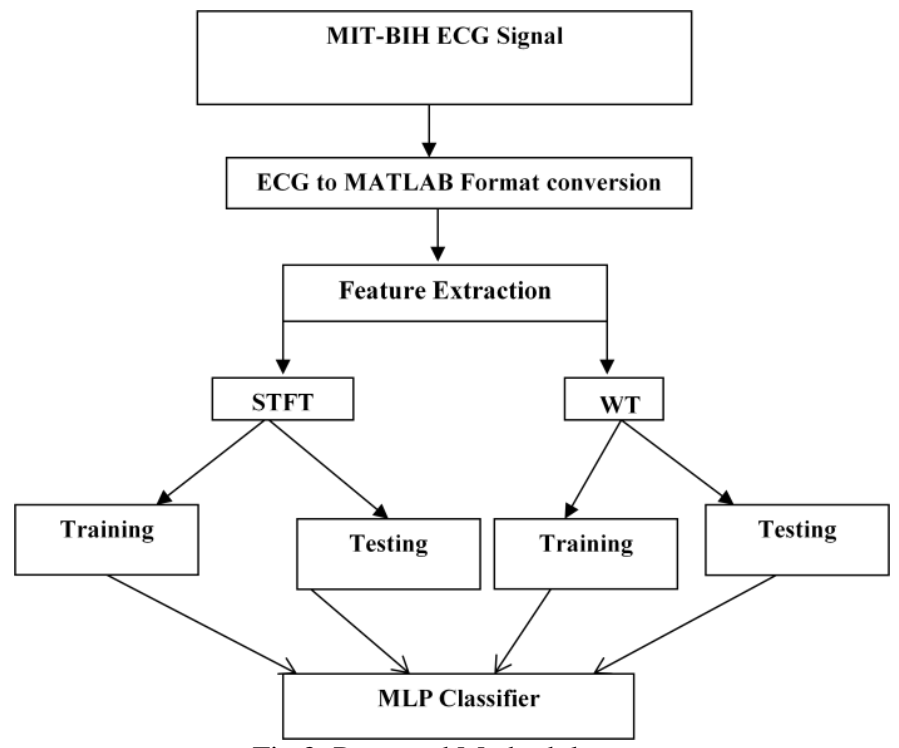

Fig.2. Proposed Methodology

Feature Extraction: Extraction of reliable feature is paramount in signal and pattern recognition as these are the minimal units that represent a maximally close class. The work uses two mostly informative spectral features such as the STFT and the DWT in classification of Arrhythmia disease in patients. The details of these two feature extraction techniques have been given below.

Short Time Fourier Transform (STFT): The Discrete Time Fourier Transform (DTFT) provides the spectrum of a digitized signal. The DFT for the sequence $s(n)$ of length $N$ is obtained by sampling the FT at $N$ discrete frequencies and is expressed as in(1):

$$
S(k)=\sum_{n=0}^{N-1} S(n) e^{-j 2 \pi k n / N}
$$

where $k$ is the frequency points. However, the spectral content of a non-stationary signal such a speech, music, ECG etc. change over time. Thus, over a the signal, application of DFT may not provide the relevant spectral transitions. Hence these signals are represented over short frame or windows by making a trade-off between time and frequency of the signal. A window size of $30 \mathrm{~ms}$ with a window overlap of $50 \%$ has been used in this work [10]. To obtain the desired STFT, the FFT is applied to each window $w$ of the signal. The discrete STFT of the signal can be expressed as in (2):

$$
S(n, k)=\sum_{m=-\infty}^{\infty} S(n, \omega), \omega=\frac{2 \pi}{k} n
$$

here $S(n, \omega)$ is the windowed FT of signal and is given by

$S(n, \omega)=\sum_{m=-\infty}^{\infty} s[m] w[n-m] e^{-j \omega n}$

Discrete Wavelet Transform $(D W T)$ : Due to availability of redundant coefficients in Continuous Wavelet Transform (CWT) signal analysis restoration of the original signal remains complex. DWT uses adaptive resolution and filter banks in describing a signal hence provides enhanced calculation efficiency. The STFT used fixed windows with uniform resolution for a signal, hence remains inferior to DWT for signal analysis as the later uses varying windows. It explores the multi-resolution capability in signal representation and hence is more versatile. It decomposes the signal into detailed $d_{y}$ and approximation coefficient $a_{y}$ by correspondingly high and low pass filtering the signal. The signal in wavelet analysis can be represented as in(4):

$s(t)=\sum_{k} a_{y}(i, \tau) \beta_{i, k}(t)+\sum_{i=1}^{I} \sum_{k} d_{y}(i, \tau) \lambda_{i, k}(t)$

In this case $\beta_{i, k}$ is the scaling coefficients in the nested space $W_{i}$ and the term $\lambda_{i, k}$ denotes the mother wavelet. The Coefficients $d_{y}$ indicates the information loss between the approximation coefficients transit and hence are more relevant as signal features. The detailed coefficients used in this work can be described as in (5):

$d_{i}(t)=a_{i-1}(t)-a_{i}(t)$

The generalized structure of DWT decomposition has been given in Fig.3. 


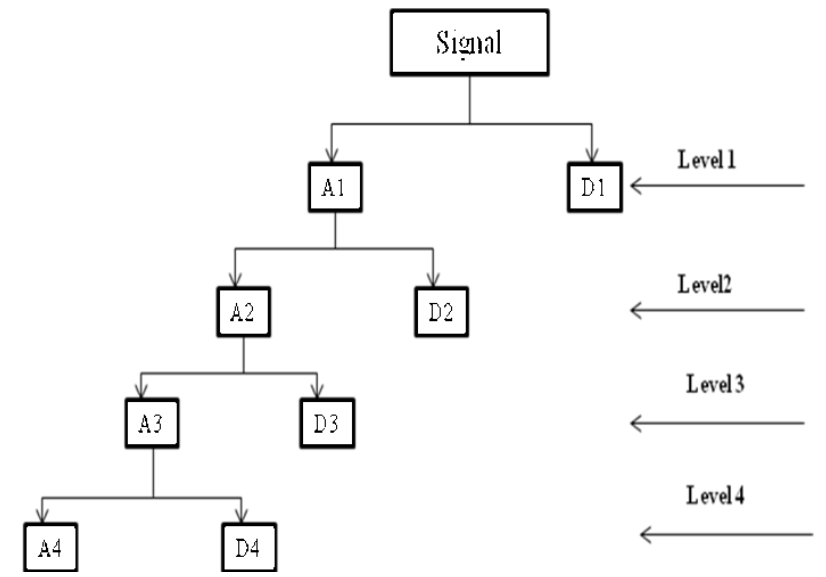

Fig.3. Generalized DWT structure for feature extraction

Classification Model: There have been a host of efficient classifiers used in the field of bio-medical signal analysis each have their limitations and advantages. This work uses the standard NN based MLP classifier for the reason explained in section I. The aim is to classify and compare the accuracy and Mean Square Error performance using two of the mostly used adaptive learning functions such as Gradient Descent (GD) and Gradient Descent with Momentum (GDM). The MLP is uses the back-propagation algorithm while updating weights and biases [11-12]. It contains input, hidden and the output layers as three major units as exposed in Fig.4.

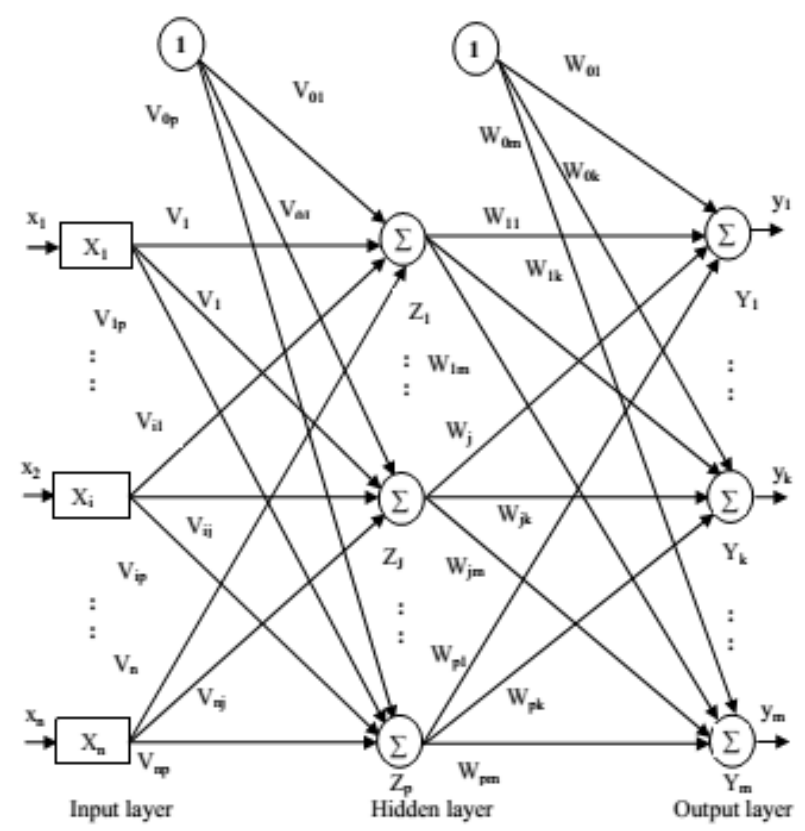

Fig.4. Generalized MLP Structure

The two adaptive learning algorithms has been explained as below

Gradient Descent Algorithm(GD): It is the first order optimization algorithm that finds a function's minimum when the negative value is taken. The maximum value of the function can be found using the positive gradient. The method can work in any feature space irrespective of its dimension and uses much iteration for computation of local minimum to obtain the desired accuracy.

It calculates the change in weight $d w$ for a known neuron from the neuron's input $P$, error $E$, the weight (or bias) and the learning rate $\alpha$ using the gradient descent, where

$d w=\alpha^{*} g_{w}$

and $g_{w}$ denotes the performance gradient. However, the algorithm is slow and converges asymptotically to the minimum. Further, it is ill-defined for non-differentiable functions and appears in zigzag manner towards for convex problems.

Gradient Descent with momentum Algorithm (GDM): For navigating ravines or steeply curves in one dimension with respect to the other that are commonly appear around local minima, the GD oscillates around the slopes. Thus the learning progresses hesitantly to attain the desired convergence. The GD with moment accelerates the GD in the direction of relevance by dampening the oscillation. Hence the method founds to be more optimal as compared to the GD method of learning [13].

The GDM calculates the change in weight $d w$ for a given neuron from the input neuron $P, E$ is the error rate, $W$ is the weight of the neurons, $\alpha$ is the rate of learning and $m$ represent the momentum constant according to gradient. The prior weight transform $d w_{p r v}$ is stored and interpret from the learning state.

$$
d w=m * d w_{p r v}+(1-m) * \alpha * g_{w}
$$

\section{RESULT DISCUSSION}

The data is separated into training, validation and testing ratios of $70 \% / 15 \% / 15 \%$ as default values of $\mathrm{NN}$ tool box used in this work. Ten numbers of hidden neurons in a hidden layers has been taken. A single hidden layer with a learning rate of 0.01 has been used for this purpose. The network is classified the signals into normal and Arrhythmia.

Decomposition of ECG Signal using DWT: The Arrhythmia signals are obtained from the MIT-BIH data base. Decomposed of the signal into approximation and the details has been done using DWT. A sampling rate of $650 \mathrm{~Hz}$ has been used for this purpose.

Different mother wavelets as DB1, DB2, DB3 and DB4 have been considered for measuring the accuracy. However, the DB4 has provided the highest classification and hence is chosen for this work. Table I provides the performance parameters of MLP using STFT and DWT features with respect to the GD and GDM adaptive learning algorithms. 
Table I: Performance parameters of MLP using STFT and DWT features

\begin{tabular}{|c|c|c|c|c|}
\hline \multicolumn{4}{|c|}{$\begin{array}{c}\text { Comparison of MLP Performance with Different Features and Adaptive } \\
\text { Learning Algorithms }\end{array}$} \\
\cline { 2 - 5 } & \multicolumn{4}{|c|}{ Features } \\
\hline $\begin{array}{c}\text { Performance } \\
\text { Parameters }\end{array}$ & GDM & GD & GDM & GD \\
\hline $\begin{array}{c}\text { Adaptive Learning } \\
\text { Function }\end{array}$ & 0.240 & 0.246 & 0.0012 & 0.0033 \\
\hline MSE & 45 & 9 & 130 & 25 \\
\hline NO. of Epochs chosen & 42 & 3 & 121 & 21 \\
\hline $\begin{array}{c}\text { Epochs at Best Validation } \\
\text { Performance }\end{array}$ & & & & \\
\hline
\end{tabular}

In this work a comparison is made between two popular spectral features such DWT and STFT to recognize the Arrhythmia disease of patient by using MLP. It has been observed that DWT has outperformed the STFT in describing the disease better. Similarly between the GDM and GD learning algorithm, the GDM has shown better accuracy as compared to the GD algorithm for both the chosen feature sets. A highest recognition performance of $80 \%$ has been experienced for the DWT feature sets with testing data sets using GDM algorithm. As compared to this, highest recognition accuracy of $53.2 \%$ has been observed for the GD algorithm with STFT feature sets. In Table II the classification accuracy has been presented.

Table II. MLP classification accuracy

\begin{tabular}{|c|c|c|c|c|c|}
\hline \multicolumn{5}{|c|}{ Comparison of MLP Accuracy with Different Data Division } \\
\hline $\begin{array}{c}\text { Featur } \\
\text { es }\end{array}$ & $\begin{array}{c}\text { Adaptive } \\
\text { learning } \\
\text { algorithm }\end{array}$ & Training & Validation & Testing & Overall \\
\hline WT & GDM & $76 \%$ & $74.4 \%$ & $80 \%$ & $76.7 \%$ \\
& GD & $59 \%$ & $63.9 \%$ & $61.7 \%$ & $60.8 \%$ \\
\hline STFT & GDM & $50.6 \%$ & $50.8 \%$ & $51.3 \%$ & $50.7 \%$ \\
& GD & $49.9 \%$ & $50.6 \%$ & $53.2 \%$ & $50.5 \%$ \\
\hline
\end{tabular}

\section{Conclusion}

Arrhythmia founds to be fatal disease hence, requires the accurate prediction and monitoring. This work attempts to classify this disease using different adaptive learning algorithms of MLP using two mostly popular spectral feature sets. The DWT with GMD algorithm of MLP has shown to outperform in the current scenario as compared to the STFT feature sets. Further efficient features and similarly effective classifiers may open up new avenues in this direction.

\section{REFERENCES}

[1] G C. S. Dangare, and S. S. Apte, "Improved study of heart disease prediction system using data mining classification techniques. International Journal of Computer Applications", Vol.47, No.10, pp.44-48, 2012.

[2] M. Gandhi, and S. N. Singh, "Predictions in heart disease using techniques of data mining", In Futuristic Trends on Computational nalysis and Knowledge Management (ABLAZE), 2015 International Conference IEEE, pp.520-525, Feb. 2015.

[3] Z. Wu, X. Ding, G. Zhang, X. Xu, X. Wang, Y. Tao, and C. Ju, "A novel features learning method for ECG arrhythmias using deep belief networks". In Digital Home (ICDH), IEE2016 6th International Conference, pp.192-196, Dec. 2016.

[4] P. Chazal, M. O'Dwyer, and R. B. Reilly, "Automatic classification of heartbeats using ECG morphology and heartbeat interval features", IEEE Transactions on Biomedical Engineering, Vol.51, No.7, pp.1196-1206, 2004.

[5] O. T. Inan, L. Giovangrandi, and G. T. Kovacs, "Robust neuralnetwork-based classification of premature ventricular contractions using wavelet transform and timing interval features" IEEE Transactions on Biomedical Engineering, Vol.53, No.12, pp.25072515,2006

[6] R. J. Martis, U. R. Acharya, and L. C. Min, "ECG beat classification using PCA, LDA, ICA and discrete wavelet transform", Biomedical Signal Processing and Control, Vol.8, No.5, pp.437-448, 2013.

[7] A. Kampouraki, G. Manis, and C. Nikou, "Heartbeat time series classification with support vector machines", IEEE Transactions on Information Technology in Biomedicine, Vol.13, No.4, pp.512-518, 2009.

[8] M. K. Gautam, and V. K. Giri, March. "A Neural Network approach and Wavelet analysis for ECG classification" In Engineering and Technology (ICETECH), IEEE International Conference on, pp. 11361141,2016,

[9] L. T. M. Thuy, N. T. Nghia, D. V. Binh, N. T. Hai, and N. M. Hung, "Error-rate analysis for ECG classification in diversity scenario", In System Science and Engineering (ICSSE),International Conference on, IEEE, pp. 39-43, Jul. 2017.

[10] H. K. Palo, M. N. Mohanty, "Wavelet based feature combination for recognition of emotions," Ain Shams Engineering Journal, Jan 2017.

[11] H. K. Palo, M. Chandra, M. N. Mohanty, "Emotion recognition using MLP and GMM for Oriya language", International Journal of Computational Vision and Robotics. No.7,Vol.4, pp. 426-42, 2017.

[12] R. D. Raut, and S. V. Dudul, "Arrhythmias classification with MLP neural network and statistical analysis," In Emerging Trends in Engineering and Technology, International Conference on , IEEE, pp. 553-558, 2008.

[13] C. Burges, T. Shaked, E. Renshaw, A. Lazier, M. Deeds, N. Hamilton, and G. Hullender, "Learning to rank using gradient descent", In Proceedings of the 22nd international conference on Machine learning, ACM, pp. 89-96,2005. 